Health Psychol. 2014 July ; 33(7): 681-685. doi:10.1037/a0035405.

\title{
Fluctuations in Depression and Well-Being Are Associated with Sexual Risk Episodes Among HIV-Positive Men
}

\author{
Patrick A. Wilson, Gertraud Stadler, Melissa R. Boone, and Niall Bolger \\ Columbia University, New York, NY
}

\section{Abstract}

Objective-Research has suggested a weak association between depression and sexual risk behavior in men who have sex with men (MSM). The purpose of this study is to investigate the relationship between within-person fluctuations in depression and well-being and episodes of sexual risk-taking among HIV-positive MSM.

Methods-One hundred and six sexually active HIV-positive MSM living in New York City completed a structured weekly survey over six weeks. In weeks 1,3 , and 5 , they responded to items assessing their sexual behavior, depression and well-being in the prior week. Multi-level logistic regression analyses were conducted to explore the relationship between changes in levels of depression and well-being and episodes of unprotected anal intercourse (UAI).

Results-Within-person deviations from participants' average levels of depression and wellbeing were associated with the probability of risk. The probability of a risk episode was higher in weeks when depression was higher than participants' average levels (any UAI episode: $O R=1.71$, 95\% CI [1.00-2.90], $p<.05$; serodiscordant UAI episode: $O R=2.49,95 \%$ CI [1.31-4.73], $p<$. $05)$. The probability of a risk episode was lower in weeks when well-being was higher than participants' average levels (any UAI: $O R=0.40,95 \%$ CI [0.22-0.74], $p<.05$; serodiscordant UAI: $O R=0.42,95 \%$ CI $[0.22-0.81], p<.05)$. Between-person differences in depression and well-being were not associated with risk episodes (all $p \mathrm{~s}>.05$ ).

Conclusions-This study is among the first to examine the association of within-person changes in depression and well-being with sexual risk behavior in a diverse sample. It contributes new evidence to literature exploring the relationship between depression and sexual risk. Future research should employ longitudinal designs to explore pathways linking within-person changes in depression with risk behavior.

\section{Keywords}

depression; well-being; HIV/AIDS; men who have sex with men; sexual risk behavior

Correspondence concerning this article should be addressed to: Patrick A. Wilson, Department of Sociomedical Sciences, Columbia University Mailman School of Public Health, 722 W. $168^{\text {th }}$ Street, New York, NY 10032. Phone: 212-305-1852; fax: 212-342-1285; pw2219@columbia.edu.

Patrick A. Wilson, Department of Sociomedical Sciences, Columbia University Mailman School of Public Health; Gertraud Stadler, Department of Psychology, Columbia University; Melissa R. Boone, Department of Sociomedical Sciences, Columbia University

Mailman School of Public Health; Niall Bolger, Department of Psychology, Columbia University. 
Previous studies exploring the relationship between depression and sexual risk behavior have produced weak evidence for an association between the two variables. A meta-analysis by Crepaz and Marks (2001) showed limited support for a positive relationship between depression and sexual risk behavior across 27 studies, each including quantitative measures of depression, anxiety, and/or anger and sexual risk behaviors associated with HIV transmission (i.e., unprotected sex, number of sex partners). Effect sizes ranging from 0.04 to 0.10 were observed. A second meta-analysis by Crepaz and Marks (2002) showed little evidence that emotional distress was associated with sexual risk behavior in HIV-positive men and women.

Methodological problems plague many of the studies exploring a link between depression and sexual risk behavior, and findings should be considered inconclusive (Kalichman \& Weinhardt, 2001; Sikkema et al., 2010). Most studies have used trait measures of depression and risk administered at one point in time to examine between-person differences in depression and risk. These studies can only address the question of whether people who have high levels of depression also exhibit high levels of sexual risk behavior; they cannot tell us about within-person changes in depression and their associations with risk. Understanding this phenomenon will allow for the development of tailored interventions that may alleviate negative affect and its sequelae.

For people living with HIV (PLWH) and men who have sex with men (MSM), depression and psychological well-being change over time (Perdue, Hagan, Thiede, \& Valleroy, 2003). These changes may be pronounced for HIV-positive MSM who are racial/ethnic minorities and of low socioeconomic status (SES). Unique psychosocial and contextual factors that affect these populations including coping with minority stress, can strengthen a potential relationship between depression and sexual risk behavior (Hatzenbuehler, O'Cleirigh, Mayer, Mimiaga, \& Safren, 2011).

The current study examines the relationship between changes in depression and well-being and episodes of sexual risk behaviors among racially diverse HIV-positive MSM. This study is designed to differentiate between- and within-person associations of depression and risk (e.g., Cooper, 2010; Shrier et al., 2011). It builds upon extant research by conceptualizing depression and well-being as dynamic variables that change over time. It is hypothesized that within-person changes in depression and well-being are related to episodes of sexual risk taking among HIV-positive MSM. In accordance with Crepaz and Marks' (2001, 2002) findings, we expect to find little evidence of a between-person association of depression and increased sexual risk behavior.

\section{Methods}

\section{Participants \& Procedures}

Participants comprised a convenience sample of HIV-positive MSM living in New York City. Recruitment took place between 2007 and 2009; 158 participants were recruited via fliers placed in neighborhood venues (i.e., coffee shops, bars) and community-based organizations (48\%), participant referrals (39\%), and media advertisements (13\%). Interested participants were screened for self-reported sexual behavior with another man in 
the past two months and were HIV-positive, as confirmed through review of medical documentation. Those eligible signed on to a secure website each week to complete a 6week Internet-based survey; data collected in weeks 1, 3, and 5 are used in the current study. Men who did not complete at least one assessment in weeks 1,3 , or $5(N=14)$ or who did not report sexual behavior during these three assessment occasions $(N=38)$ were excluded from the analysis. Participants that were excluded did not differ from those included in the final sample on key variables. The average age of participants was 38.3 years $(S D=9.7)$. Approximately half (51.9\%) of participants were African-American, while $25.5 \%$ were Latino/Hispanic, $20.8 \%$ were White, and $1.9 \%$ were "Other" race. The sample could be described as low SES: $69.5 \%$ of participants reported an income of $\$ 20,000 /$ year or less and only $32.3 \%$ were employed or a student. Most (82\%) identified as gay/homosexual and $81 \%$ reported that they were currently having sex with multiple partners. Sixty-six percent reported UAI in the prior two months. Detailed sample information can be found in Table A1 in Appendix A of the Supplemental Digital Content file. All procedures were IRBapproved (CUMC IRB \#AAAC3151).

\section{Measures}

To assess sexual risk episodes, participants were asked each week in the survey if they had a sexual encounter that week. Those answering yes were asked to report on their last sexual encounter and what sexual behaviors they engaged in, including UAI. They were also asked to indicate the HIV status and gender of their sex partner(s). Sexual episodes $(n=191)$ were categorized according to whether UAI occurred (i.e., "any UAI episode;" $n=107$, reported by $64 \%$ of participants) and whether UAI occurred with an HIV-negative or unknown status partner (i.e., "serodiscordant UAI episode;" $n=33$, reported by $24 \%$ of participants). The majority (95.8\%) of sexual episodes occurred with male partners, while $1.6 \%$ and $2.6 \%$ occurred with female and transgender partners, respectively.

Measures of depression and well-being were administered in Weeks 1, 3, and 5 of the study, including the Centers for Epidemiological Studies-Depression (CES-D; Radloff, 1977) scale and the Functional Assessment of HIV Infection (FAHI; Peterman, Cella, Mo, \& McCain, 1997). The CES-D is a 20-item scale widely used to assess depressed affect in diverse populations. The reliability for detecting within-person systematic change in depression from week to week was high in the sample, $R_{\text {Change }}=0.93$, as was the reliability for detecting stable between-person differences in depression across weeks, $R_{\text {Betw }}=0.94$. The FAHI is used to assess well-being in PLWH. Three subscales comprised of 28 items measuring physical, social, and emotional well-being were used to form a general wellbeing index. The FAHI's reliability for detecting within-person change in well-being from week to week was high, $R_{\text {Change }}=0.96$, as was the reliability for detecting stable betweenperson differences in well-being across weeks, $R_{\text {Betw }}=0.98$. See Appendix B of the Supplemental Digital Content file for details regarding reliability calculations.

\section{Analysis}

Our study design allowed for a contemporaneous within-person analysis (Bolger, Davis, \& Rafaeli, 2003) of the relationship between depression and UAI at weeks 1, 3, and 5. A contemporaneous analysis approach was chosen over a time-lagged approach because the 2 - 
week lags between assessment occasions were not able to capture processes that would be expected to play out in hours or days (Remien, Carballo-Dièguez, \& Wagner, 1995; Wilson, Cook, \& Valera, 2013). Accordingly, within-person analyses were conducted to test the hypothesis that on weeks when depression was higher and well-being was lower than a participant's average, he would have a higher likelihood of having a UAI episode.

$$
\ln \frac{\operatorname{Pr}\left(U A I_{i t}=1\right)}{\operatorname{Pr}\left(U A I_{i t}=0\right)}=\beta_{1}+\beta_{2} \text { Depression }_{-} W_{i t}+\beta_{3} \text { Depression }_{-} B_{i}
$$

Equation 1 illustrates the data analysis approach for probability of UAI and depression. To test our hypotheses, we modeled the probability of a risk episode at weeks 1,3 , and 5 as a logistic function with two predictors: (a) deviations in depression for person $i$ at time $t$ (Depression_ $W_{i t}$ ) from the person mean level of depression, and (b) between-person mean levels of depression, centered at the grand mean (Depression_ $B_{i}$,) (Raudenbush \& Bryk, 2002; Curran \& Bauer, 2011). Within-person deviations in depression and person means were divided by the between-person standard deviation to facilitate interpretation of effects. The coefficient $\beta_{2}$ tests whether on weeks when a participant was more depressed than usual he was more likely to engage in a risk episode than usual (within-person association); the coefficient $\beta_{3}$ tests whether men who were higher in average depression were also higher in average risk (between-person association). Identical analyses were carried out examining within- and between-person levels of well-being as predictors of risk episodes. Analyses were carried out using PROC GENMOD in SAS 9.3. We report unadjusted estimates ${ }^{1}$ and employ a significance level of $p \leq .05$ (see Appendix C in the Supplemental Digital Content file for a detailed description).

\section{Results}

Of the 106 participants who were included in the analysis, 44, 39, and 23 participants completed one, two, and three assessments, respectively. The person mean for depression across the time points was $16.41(S D=10.19$, on a scale from 0 to 60$)$, with $41.5 \%$ of participants scoring 16 or higher, the cutoff for elevated depression. The person mean for well-being across the three time points was 25.99 ( $S D=6.52$, on a scale from 0 to 39 ).

The probability of any UAI episode was higher when depression was greater than usual ( $O R$ $=1.71,95 \% \mathrm{CI}[1.00-2.90], p<.05)$ and when well-being was lower than usual $(O R=0.40$, 95\% CI [0.22-0.74], $p<.05)$. Panels A and B of Figure 1 show that when well-being and depression were at average levels, a participant had a 56\% probability of having any UAI episode. For weeks when a participant's depression was $-1 S D(10.19)$ lower than usual, the probability of any UAI episode was $43 \%$; when depression was $+1 S D$ higher than usual), the probability was $69 \%$ (Panel A). Similarly, when a participant's well-being was $+1 S D$ (6.52) higher than usual, the probability of any UAI risk episode was $34 \%$; when it was -1

\footnotetext{
${ }^{1}$ Sensitivity analyses were conducted using separate models that included demographic and health variables commonly used research exploring sexual risk behavior. Across multiple models, the pattern of results remained the same: within-person associations were statistically significant, but between-person associations between depression/well-being and risk were not. In the interest of parsimony unadjusted models are reported.
} 
$S D$ (6.52) lower than usual, the probability was 76\% (Panel B). Between-person differences in depression and well-being were shown to be nonsignificant (depression: $O R=1.25,95 \%$ CI [0.86-1.80], $p=.24$; well-being: $O R=0.75,95 \%$ CI [0.50-1.11], $p=.15$ ).

The probability of a serodiscordant UAI episode was higher when depression levels were greater than usual $(O R=2.49,95 \% \mathrm{CI}[1.31-4.73], p<.05)$ and when well-being was lower than usual $(O R=0.42,95 \%$ CI [0.22-0.81], $p<.05)$. Panels C and D of Figure 1 show that when well-being and depression were at average levels, a participant had a 17\% probability of having a serodiscordant UAI episode. When a participant's depression was $-1 S D$ (10.19) lower than usual, the probability for a serodiscordant UAI episode was 7\%; when depression was $+1 S D$ higher than usual, the probability was $33 \%$ (Panel C). Similarly, for weeks when a participant's well-being was $+1 S D(6.52)$ points higher than usual the probability for a serodiscordant UAI episode was $8 \%$; when well-being was $-1 S D$ lower than usual the probability was $32 \%$ (Panel D). Between-person differences in depression and well-being were nonsignificant (depression: $O R=1.01,95 \% \mathrm{CI}$ [0.66-1.57], $p=.95$; well-being: $O R=$ $0.95,95 \%$ CI [0.63-1.42], $p=.79$ ). Table C.2 (in Appendix C of the Supplemental Digital Content file) provides the results in detail.

\section{Discussion}

Within-person associations between depression and well-being, and sexual risk episodes, were observed in a racially diverse sample of sexually active HIV-positive MSM. During times when participants felt less depression and greater well-being than usual, risk episodes were less likely to occur; during weeks when they felt more depression and less well-being than usual, risk episodes were more likely to occur. Between-person associations of wellbeing and risk were not supported.

Our findings help explain why previous research has found inconsistent support for a link between depression and sexual risk behavior. This study provides preliminary evidence that, for many HIV-positive MSM, this relationship exists at the within-person level. Studies that utilize between-person designs and trait measures - the majority of studies in this area (Kalichman \& Weinhardt, 2001) — are not equipped to detect this relationship. Stable individual and contextual factors cannot explain the findings, as participants show less sexual risk behavior during weeks when they feel less depressed and more risk behavior during weeks when they feel more depressed.

Although evidence of within-person associations of changes in depression and well-being with sexual risk episodes is provided in this research, cause and effect are not fully clear. Strong evidence for the depression-to-risk relationship comes from intervention studies with MSM and PLWH, which have found that improving mental health leads to reduced sexual risk behavior (Sikkema et al, 2008; 2010). Increases in depression and decreases in wellbeing among sexually active MSM may result in externalizing symptoms, such as sensationseeking and the use of sex to avoid or alleviate negative affect, as opposed to internalizing symptoms, such as withdrawal (Alvy et al., 2011; Mustanski, 2007); these externalizing symptoms may lead to sexual risk-taking. Indeed, studies have suggested that moderately 
depressed individuals may regulate negative affective states by engaging in sexual behaviors (Reisner et al., 2009; Shrier et al., 2011).

Despite strong evidence for the depression-to-risk path, changes in sexual risk behavior may also lead to changes in depression and well-being. When HIV-positive MSM engage in behavior that may put a partner at risk, they may feel regret or shame (O'Leary \& Wolitski, 2009; Remien, Carballo-Dièguez, \& Wagner, 1995; Wilson, Cook, \& Valera, 2013), and these feelings may lead to decreased well-being. However, the few prospective studies exploring the relationship between affect and sexual risk behavior provide little evidence to substantiate the risk-to-depression path (Cooper, 2010; Mustanski, 2007). Nonetheless, bidirectional causality is plausible for a within-person link between depression and sexual risk behavior. Further research for explaining the within-person association will require longitudinal studies capturing time-varying process variables, such as substance use and daily stressors, in addition to psychometrically robust measures of depression and sexual risk behavior.

Our study is limited in that the data collected do not allow for the specification of how factors such as emotion regulation and other within-person processes, such as changes in receipt of social support, may mediate or moderate the observed association of fluctuations in well-being with sexual risk behavior over time. Other limitations include use of a convenience sample of HIV-positive MSM, which reduces generalizability, and the number of assessment occasions, which limited the ability to conduct cross-lagged analyses and describe causal directionality.

Despite these limitations, this study represents a critical step forward in describing the complexities of the relationship between well-being and sexual risk behavior. Future research should obtain more fine-grained data to examine stressors that are related to negative affect and may prompt engagement in sexual risk behavior as a way to regulate emotions. Race, sexual orientation, and HIV related stressors might have influenced mental health in our sample of racially diverse, low-income HIV-positive MSM (Hatzenbuehler, O'Cleirigh, Mayer, Mimiaga, \& Safren, 2011; Meyer, 2010). Mental health interventions that aim to improve well-being and enhance healthful emotion regulation and coping behaviors are warranted. In addition to improving overall mental health among HIV-positive MSM, these interventions have the potential to reduce sexual risk-taking behaviors that promote the transmission of HIV and other sexually transmitted infections.

\section{Supplementary Material}

Refer to Web version on PubMed Central for supplementary material.

\section{Acknowledgments}

This research was supported by the Center for Interdisciplinary Research on AIDS (CIRA) at Yale University through a grant from the National Institute of Mental Health (P30 MH 62294, awarded to Mike Merson, M.D.). The work was also supported by a development grant from the Office of the Vice Provost for Diversity Initiatives, Columbia University. 


\section{References}

Alvy LM, McKirnan DJ, Mansergh G, Koblin B, Colfax GN, Flores SA, et al. Depression is associated with sexual risk among men who have sex with men, but is mediated by cognitive escape and self efficacy. AIDS and Behavior. 2011; 15:1171-1179. [PubMed: 20217471]

Cooper ML. Toward a person x situation model of sexual risk-taking behaviors: Illuminating the conditional effects of traits across sexual situations and relationship contexts. Journal of Personality and Social Psychology. 2010; 98:319-341. [PubMed: 20085403]

Crepaz N, Marks G. Towards an understanding of sexual risk behavior in people living with HIV: A review of social, psychological, and medical findings. AIDS. 2002; 16:135-149. [PubMed: 11807297]

Crepaz N, Marks G. Are negative affective states associated with HIV sexual risk behaviors? A metaanalytic review. Health Psychology. 2001; 20:291-299. [PubMed: 11515741]

Curran PJ, Bauer DJ. The disaggregation of within-person and between-person effects in longitudinal models of change. Annual Review of Psychology. 2011; 62:583-619.

Hatzenbuehler ML, O'Cleirigh C, Mayer KH, Mimiaga MJ, Safren SA. Prospective associations between HIV-related stigma, transmission risk behaviors, and adverse mental health outcomes in men who have sex with men. Annals of Behavioral Medicine. 2011; 42:227-234. [PubMed: 21533623]

Kalichman SC, Weinhardt L. Negative affect and sexual risk behavior: Comment on Crepaz and Marks (2001). Health Psychology. 2001; 20:300-301. [PubMed: 11515742]

Mustanski B. The influence of state and trait affect on HIV risk behaviors: a daily diary study of MSM. Health Psychology. 2007; 26:618-626. [PubMed: 17845113]

O'Leary A, Wolitski RJ. Moral agency and the sexual transmission of HIV. Psychological Bulletin. 2009; 135:478-494. [PubMed: 19379026]

Perdue T, Hagan H, Thiede H, Valleroy L. Depression and HIV risk behavior among Seattle-area injection drug users and young men who have sex with men. AIDS Education and Prevention. 2003; 15:81-92. [PubMed: 12627745]

Peterman AH, Cella D, Mo F, McCain N. Psychometric validation of the revised Functional Assessment of Human Immunodeficiency Virus Infection (FAHI) quality of life instrument. Quality of Life Research. 1997; 6:572-584. [PubMed: 9330556]

Radloff LS. The CES-D scale: A self-report depression scale for research in the general population. Applied Psychological Measurement. 1977; 1:385-401.

Raudenbush, SW.; Bryk, AS. Hierarchical linear models. 2. Thousand Oaks, CA: Sage; 2002.

Reisner SL, Mimiaga MJ, Safren SA, Mayer KH. Stressful or traumatic life events, post-traumatic stress disorder (PTSD) symptoms, and HIV sexual risk taking among men who have sex with men. AIDS Care. 2009; 21:1481-1489. [PubMed: 20024727]

Remien RH, Carballo-Dièguez A, Wagner G. Intimacy and sexual risk behavior in serodiscordant male couples. AIDS Care. 1995; 7:429-438. [PubMed: 8547358]

Shrier LA, Walls C, Lops C, Kendall AD, Blood EA. Substance use, sexual intercourse, and condom nonuse among depressed adolescents and young adults. Journal of Adolescent Health. 2011; 50:264-270. [PubMed: 22325132]

Sikkema KJ, Watt MH, Drabkin AS, Meade CS, Hansen NB, Pence BW. Mental health treatment to reduce HIV transmission risk behavior: A positive prevention model. AIDS \& Behavior. 2010; 14:252-262. [PubMed: 20013043]

Sikkema KJ, Wilson PA, Hansen NB, Kochman A, Neufeld S, Ghebremichael MS, et al. Effects of a coping intervention on transmission risk behavior among people living with HIV/AIDS and a history of childhood sexual abuse. Journal of Acquired Immune Deficiency Syndrome. 2008; 47:506-513.

Wilson PA, Cook SH, Valera P. Affect and sexual encounters among HIV-positive gay men: A situation-level analysis. Archives of Sexual Behavior. 2013 Submitted to. 

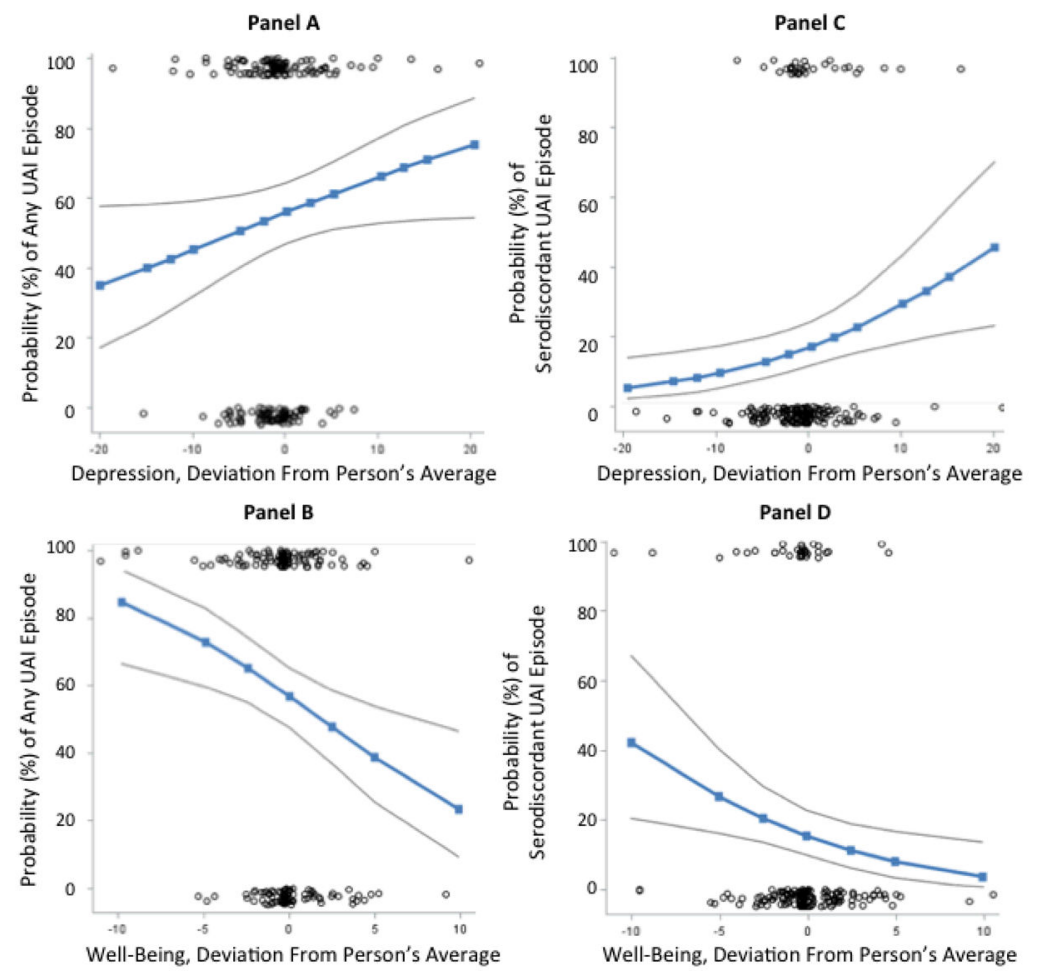

Figure 1.

Scatterplots of sexual episodes and model-based estimates of the probability of risk as a function of within-person changes in depression and well-being. Within-person deviations from a participant's average level of depression and well-being were associated with the probability of a risk episode in the past week (Panel A: depression and any UAI episode; Panel B: well-being and any UAI episode; Panel C: depression and serodiscordant UAI episodes; Panel D: well-being and serodiscordant UAI episodes). The $\mathrm{x}$ axes show deviations from each person's average in depression and well-being. Deviation scores above zero indicate weeks with higher-than-usual depression or well-being; deviation scores below zero indicate weeks with lower-than-usual depression or well-being. The y axes show the probability of any UAI episode or a serodiscordant UAI episode. Each circle indicates a sexual episode. Sexual episodes are plotted on the ends of the y axes as they were coded dichotomously to indicate the occurrence of any UAI (Panels A and B) or serodiscordant UAI (Panels C and D). The dark line in each panel indicates the estimated probability of any UAI episode or a serodiscordant UAI episode for varying deviations from a participant's average depression or well-being. The outer grey lines indicate the $95 \%$ confidence intervals around the estimated probability lines. 